\title{
Differential Expression of $\beta$-Galactoside $\alpha 2,6$ Sialyltransferase and Sialoglycans in Normal and Cirrhotic Liver and Hepatocellular Carcinoma
}

\author{
Yi Cao, Anette Merling, Paul R. Crocker, Ruprecht Keller, and \\ Reinhard Schwartz-Albiez
}

Tumor Immunology Program (YC, AM, RS-A), German Cancer Research Center, Heidelberg, and Central Laboratory (RK), Municipal Hospitals, Cologne, Germany; and The Wellcome Trust Biocentre (PRC), School of Life Sciences, University of Dundee, Dundee, Scotland

SUMMARY: Sialyltransferases sialylate plasma glycoproteins in hepatocytes and may (as hepatic key enzymes) constitute markers for liver diseases. We examined expression of the prevalent $\alpha 2,6$ sialyltransferase (ST6Gal I) and sialoglycans in normal liver, cirrhotic liver, and hepatocellular carcinoma (HCC) using a new ST6Gal I-specific mAb and recombinant fusion proteins of CD22 and sialoadhesin recognizing $\alpha 2,6$ - or $\alpha 2,3$-sialylated glycans in immunohistology and flow cytometry. In normal and cirrhotic liver, ST6Gal I and sialoglycans were localized in the Golgi region of hepatocytes surrounding the bile canaliculi and along the bile canaliculi, respectively. Sialoglycans were additionally recognized in Kupffer cells, bile ducts, endothelial cells, and oval cells. Well-differentiated and moderately differentiated HCC showed Golgi and diffuse cytoplasmic staining of ST6Gal I and sialoglycans, whereas the cytoplasmic staining for ST6Gal I and sialoglycans was decreased or even absent in poorly differentiated HCC. Detection of sialoglycans by the recombinant fusion proteins in Western blots of cell lysates derived from cell lines revealed two major double bands of sialoglycoproteins at 65 and $120 \mathrm{kDa}$ for hepatocytes, three major bands at 54 , 49, and $44 \mathrm{kDa}$ for colonic epithelial cells, and one band at $60 \mathrm{kDa}$ for endothelial cells. Our results describe the expression patterns of ST6Gal I and sialoglycans in various liver tissues and demonstrate an altered expression of these structures between benign and malignant hepatocellular lesions. (Lab Invest 2002, 82:1515-1524).

\begin{abstract}
S ialic acid attached to surface-expressed glyco$\int$ proteins or glycosphingolipids plays an important role in many cellular processes including immune recognition, binding of pathogens to host cells, cell adhesion, and apoptosis (Varki, 1999). Lectins, predominantly expressed on leukocytes, have been described that specifically recognize glycan structures sialylated in different linkages. Selectins involved in directing leukocytes to inflammatory sites require ligands with $\alpha 2,3$-linked sialic acid. Members of the siglec family either bind to $\alpha 2,6$-sialylated or $\alpha 2,3-$ sialylated glycans (Crocker and Varki, 2001). CD22 (Siglec-3) is a B-cell specific differentiation antigen involved in the regulation of $B$-cell activation and binds to $\alpha 2,6$-sialylated lactosaminyl (Gal $\beta 1,4 \mathrm{GlcNAc}$ ) sequences (Sgroi et al, 1993); sialoadhesin (Siglec-1, CD169) is expressed on distinct populations of macrophages and binds to $\alpha 2,3$-linked sialoglycans (Hartnell et al, 2001).
\end{abstract}

\section{DOI: 10.1097/01.LAB.0000038503.34655.98}

Received June 19, 2002.

The study was supported by Grant SCH 381/4-1 from the Deutsche Forschungsgemeinschaft (to RSA).

Address reprint requests to: Dr. R. Schwartz-Albiez, Division of Cellular Immunology, German Cancer Research Centre, Im Neuenheimer Feld 280,D-69120 Heidelberg, Germany.E-mail: r.s-albiez@dkfz.de
Also several human diseases, in particular cancer, are associated with aberrant expression of sialoglycans (Vierbuchen et al, 1995). Their functional influence on the development of tumors, in particular with regard to their binding to specific lectins on host cells, is still unclear.

Sialyltransferases are the key enzymes that regulate the generation of sialoglycans. Sialyltransferases consist of a family of glycosyltransferases that transfer sialic acid from the donor substrate CMP-sialic acid to different acceptor oligosaccharide substrates (Tsuji et al, 1996). The $\beta$-galactoside $\alpha 2,6$ sialyltransferase (ST6Gal I) sialylates lactosaminyl termini of complextype oligosaccharides in $\alpha 2,6$ linkage, which commonly occur on serum glycoproteins and on many cell surface glycoproteins. ST6Gal I is located predominantly in the trans-Golgi network (Roth et al, 1985) and has been identified as a soluble product after proteolytic cleavage of the membrane-spanning domain (Kitazume et al, 2001) and as a cell surface-expressed ectoenzyme (Gross et al, 1996).

In many studies expression and activity of ST6Gal I has been associated with the malignant phenotype. Colon cancer tissues display an increased activity of ST6Gal I and its $\alpha 2,6$ sialylated glycan products as compared with normal colon tissue (Dall'Olio et al, 2000). ST6Gal I activity is further correlated to the metastasizing capacity of colorectal tumor cells (Har- 
vey et al, 1992) and, in similarly, high activity of ST6Gal I in colorectal carcinomas has been associated with poor patient survival (Schneider et al, 2001).

Hepatocytes express ST6Gal I in considerable quantities (Kaneko et al, 1995) as a key enzyme for the $\alpha 2,6$ sialylation of plasma glycoproteins. ST6Gal I expression is induced as part of the acute phase response (Dalziel et al, 1999). Despite its importance for liver metabolism, our knowledge about the synthesis and expression of sialyltransferases and the respective sialoglycans in various liver diseases, in particular human hepatocellular carcinomas (HCC), is still sparse. It has been reported that ST6Gal I activity is elevated during neoplastic conditions in rat liver (Jain et al, 1993; Pousset et al, 1997).

In this study we describe expression of ST6Gal I and sialoglycans in normal and cirrhotic liver and liver tumors using a newly developed mAb against human ST6Gal I and recombinant fusion proteins of CD22 (CD22Rg) and sialoadhesin $(\mathrm{SnRg})$ to detect differently sialylated glycans. Carrier proteins of $\alpha 2,6$-sialylated and $\alpha 2,3-$ sialylated glycans in HCC cell lines were analyzed by immunoprecipitation and Western blotting. The possible usefulness of ST6Gal I and sialoglycan expression for diagnosis of liver diseases is discussed.

\section{Results}

\section{Expression and Localization of ST6Gal I and Glycotopes in Human HCC Cell Lines}

The mAb ST6-G1 raised against a defined peptide sequence of human ST6Gal I reacted specifically with the $47-\mathrm{kDa}$ band of ST6Gal I in Western blots of proteins extracted from a rat liver and human $\mathrm{HCC}$ cell lines HepG2 and HuH7 (Fig. 1A). It does not crossreact with other proteins in rat liver and human $\mathrm{HCC}$ cells and thus is well suited for studying expression and localization of ST6Gal I in liver tissues. Moreover,
$\mathbf{A}$

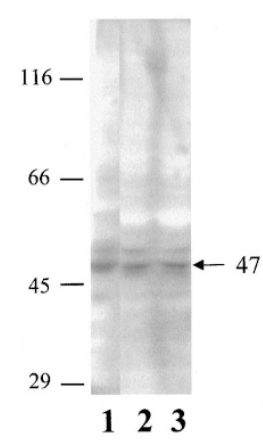

B

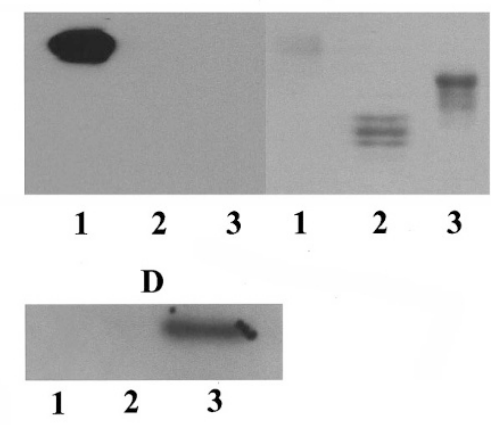

Figure 1.

Specific reactivity of mAb $\beta$-galactoside $\alpha 2,6$ sialyltransferase (ST6-G1). A, Immunoblot reaction of MAb ST6-G1 with ST6Gal I derived from rat liver (lane 1) and hepatocellular carcinoma (HCC) cell lines HuH7 (lane 2) and HepG2 (lane 3). B, Immunoblot reaction of MAb ST6-G1 with ST6Gal I from rat liver (lane1), recombinant rat ST3Gal III (lane 2), and recombinant rat ST3Gal I (lane 3). C, Amido black stains of Western-blotted sialyltransferases corresponding to immunoblots shown in B. D, Immunoblot of mAb ST6-G1 on untreated COS cells (lane 1), mock-treated COS cells with the respective expression vector pKEX-2-XR (lane 2), and ST6Gal I-transfected COS cells (lane 3).
mAb ST6-G1 did not react with other sialyltransferase such as ST3Gal I and ST3Gal III (Fig. 1, B and C) and recognized the human ST6Gal I in ST6Gal I-transfected COS cells (Fig. 1D). Untreated COS cells were negative for ST6Gal I RNA (Keppler et al, 1992).

HepG2 and HuH7 expressed ST6Gal I intracellularly, most likely in the Golgi region, as demonstrated by immunocytochemistry (Fig. 2). By flow cytometry, ST6Gal I became visible only after treatment of cells with saponin (Fig. 3). Both cell lines expressed $\alpha 2,6-$ sialylated and $\alpha 2,3-$ sialylated glycan structures at the cell surface and as Golgi constituents as evidenced by their reactivity with CD22Rg, SnRg (Figs. 2 and 3), and plant lectins Sambucus nigra agglutinin (SNA) and Maackia amurensis agglutinin II (MAA). Histo-blood group Thomsen-Friedenreich antigen (TF) was strongly expressed in its sialylated form in both cell lines in contrast to nonsialylated TF, which was detected only in $\mathrm{HuH} 7$ cells (Table 1, Fig. 3). The precursor molecule for TF, Tn was neither expressed in its sialylated nor in its nonsialylated form in both cell lines. The ganglioside GD3 was selectively expressed in its 7-O-acetylated form as shown by immune reaction with mAb U5; mAb UM4D4 against 9-O-acetyl GD3 was negative.

\section{Expression and Localization of ST6Gal I and CD22 Ligand, and Sialoadhesin Ligand in Human Normal Liver, Cirrhosis, and HCC}

The immunohistologic staining results for ST6Gal I and CD22 ligand, and sialoadhesin ligand in human donor liver and various liver diseases including cirrhosis, HCC, cholangiocellular carcinomas, and malignant epithelioid hemangioendothelioma are summarized in Table 2 and documented in Figure 2.

In normal human liver, reaction with anti-ST6Gal I $\mathrm{mAb}$ showed a granular staining characteristic of the Golgi region in normal hepatocytes surrounding the bile canaliculi but was negative in other cells such as biliary epithelial cells, endothelial cells, Kupffer cells, and Ito cells (Fig. 2D). The ligands for CD22 and sialoadhesin showed similar distribution: in hepatocytes, present along the bile canaliculi; at the apical membrane of bile ducts; and present in the cytoplasm of Kupffer cells and vascular endothelium (Fig. 2J).

In liver cirrhosis, hepatocytes were positive for ST6Gal I only in the Golgi regions as observed with normal liver (Fig. 2E). The proliferated oval cells and the pseudotubules were negative for ST6Gal I. Hepatocytes expressed CD22 ligand and sialoadhesin ligand at the apical membrane, along the bile canaliculi. Oval cells were positive for both ligands in the cytoplasm.

Instead of this regular and homogeneous distribution of ST6Gal I in normal hepatocytes and benign hepatocellular lesions, HCCs display irregularly distributed granular or lumpy stainings of ST6Gal I in the Golgi region and diffuse cytoplasm of the tumor cells (Fig. 2, F and G). The tumor cells strongly expressed ligands for CD22 and sialoadhesin in the Golgi region, in the diffuse cytoplasm, and at the plasma membrane (Fig. 2K). In poorly differentiated HCCs, staining of 

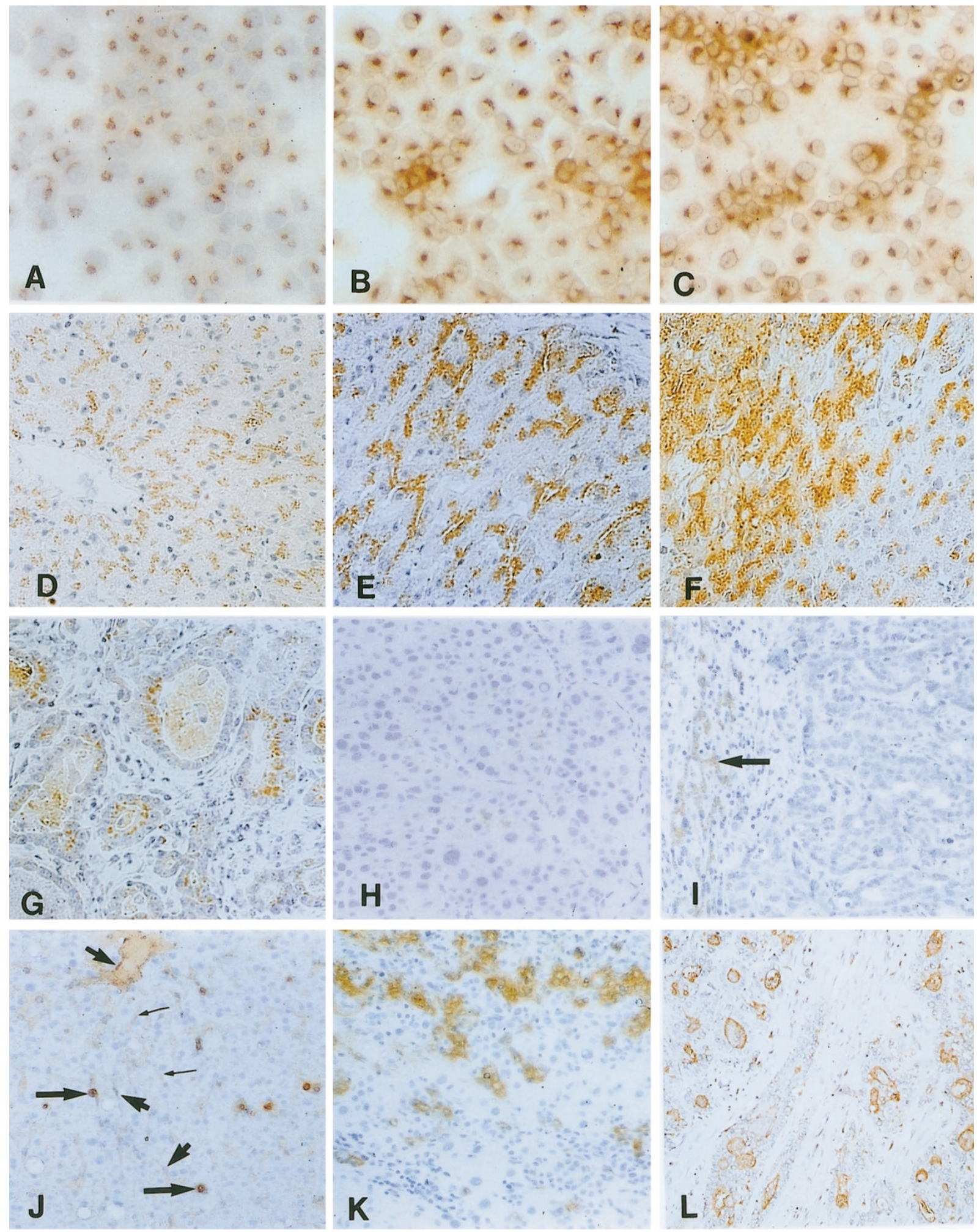

\section{Figure 2.}

Immunohistology of ST6Gal I and sialoglycans in HCCs and normal and cirrhotic liver. HuH7 cell line tumor cells are positive for ST6Gal I (A), for CD22Rg (B), and SnRg $(C)$ in the Golgi region. Some tumor cells express ligands for CD22 and sialoadhesin in the cytoplasm and at the plasma membrane. D, In normal liver hepatocytes are positive for ST6Gal I only in the Golgi region. Kupffer cells and endothelial cells are negative for ST6Gal.I. E, In liver cirrhosis, hepatocytes are positive for ST6Gal I in the Golgi region, similar to normal liver. F, In well-differentiated HCC, tumor cells express ST6Gal I in the Golgi region and cytoplasm. Some tumor cells are negative for ST6Gal I. G, In glandular formal HCC, tumor cells express ST6Gal I in the Golgi region. H, In poorly differentiated HCC, most tumor cells lose ST6Gal I. Only a few tumor cells express ST6Gal I in the cytoplasm. I, In cholangiocarcinoma, most tumor cells are negative for ST6Gal I. Hepatocytes (arrow) are positive ST6Gal I. J, In normal liver, ligands for CD22 are present along the bile canaliculi (small arrows) in hepatocytes. In Kupffer cells (big and long arrows) and endothelium (big and short arrows), CD22 ligands are present in cytoplasm. K, In HCC, tumor cells strongly express CD22 ligands in cytoplasm and at the plasma membrane. L, In cholangiocarcinoma, tumor cells strongly express CD22 ligands in cytoplasm and at the plasma membrane. 

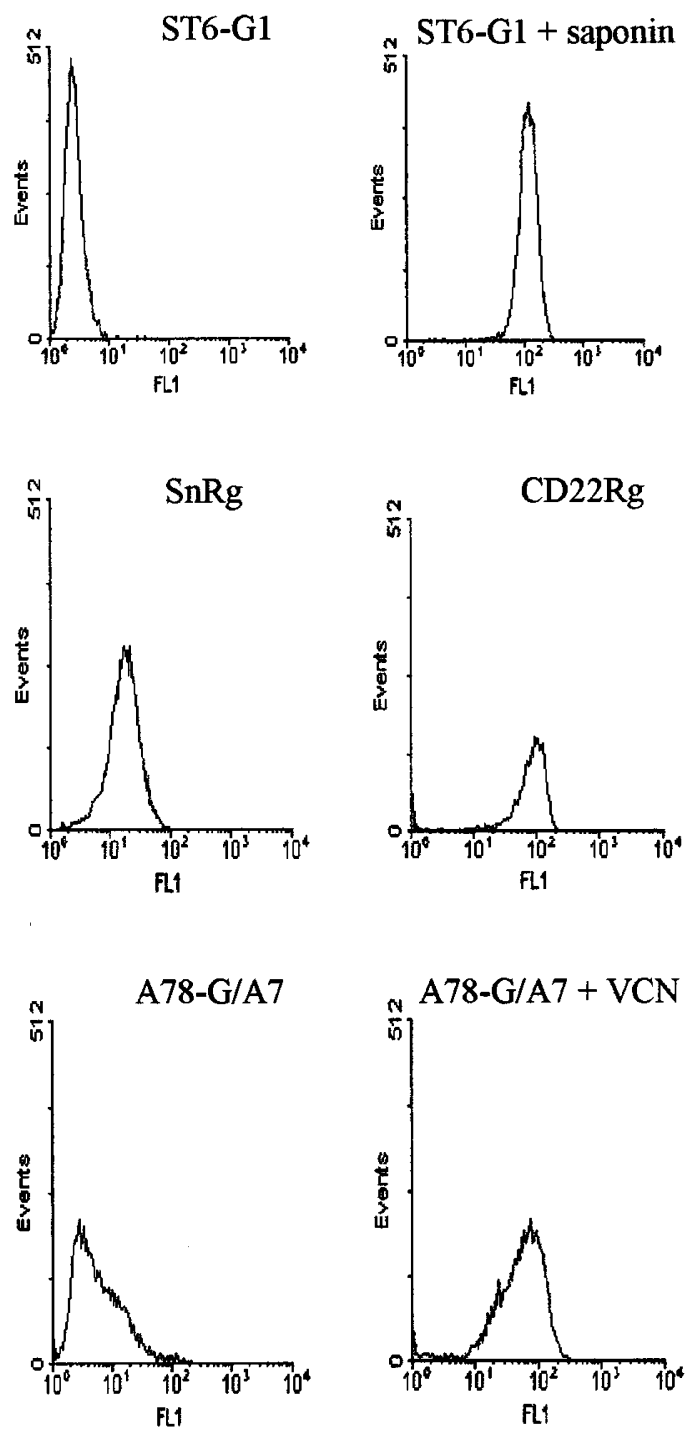

Figure 3.

Flow cytometric analysis of ST6Gal I and ligands for CD22, sialoadhesin, and sialylated TF on $\mathrm{HuH7}$. HuH7 is negative for ST6Gal I surface expression. Cellular pretreatment with saponin reveals cytoplasmic expression of ST6Gal I. $\mathrm{HuH} 7$ is positive for $\mathrm{CD} 22 \mathrm{Rg}$ and $\mathrm{SnRg}$ binding. $\mathrm{HuH} 7$ cells weakly bind $\mathrm{mAb}$ A78-G/A7 against TF and much stronger after cellular VCN treatment.

ST6Gal I was decreased to the largest extent and even disappeared. Most tumor cells also lost ligands for CD22 and sialoadhesin. Only a few tumor cells expressed ST6Gal I and ligands for CD22 and sialoadhesin in the diffuse cytoplasm (Fig. 2H).

One case each of cholangiocellular carcinoma and of malignant epithelioid hemangioendothelioma did not express ST6Gal I. In cholangiocarcinoma and endothelial tumor, tumor cells strongly expressed ligands for CD22 and sialoadhesin in the cytoplasm and at the plasma membrane (Fig. 2, I and L).

\section{Analysis of Carrier Molecules of Ligands for CD22 and Sialoadhesin in Human HCC Cell Lines}

In immunocytochemistry, staining of HepG2 and $\mathrm{HuH} 7$ with CD22Rg, SnRg, SNA, and MAA was not completely abolished after methanol extraction, indicating that glycosphingolipids are not unique carrier molecules of these sialoglycans. Western blotting revealed major bands of 120 and $65 \mathrm{kDa}$ as carrier proteins of ligands for CD22, sialoadhesin, SNA, and MAA in the HCC cell lines HepG2 and HuH2 (Fig. 4A). In contrast, the approximate molecular weights of carrier proteins of ligands for CD22 and sialoadhesin in the colon carcinoma cell lines HT-29 and CaCo-2 and in the endothelial cell line HBMEC-60 were 54, 49, and $44 \mathrm{kDa}$, respectively; additionally, for HBMEC-60, the molecular weight was $60 \mathrm{kDa}$ (Fig. 4C). Immunoprecipitation and Western blotting with neuraminidase-treated $\mathrm{HuH} 7$ showed a series of TF-carrying glycoproteins with three main bands: $>205 \mathrm{kDa}, 205 \mathrm{kDa}$, and $150 \mathrm{kDa}$ (different from those found with CD22 and sialoadhesin; Fig. 4B).

To look for sialylation of glycosphingolipids in HCC, we applied mAbs against defined gangliosides. Immunostaining for GD3 was negative. Interestingly GD3 was almost exclusively expressed in its 7-O-acetylated form in HCC cells as demonstrated by staining with $\mathrm{mAb}$ U5. Because immunostaining with mAb U5 was abolished after methanol extraction, we conclude that the 7-O-acetyl-disialoglycan sequence was exclusively expressed as the ganglioside GD3. GM3, representing the precursor sequence of GD3, was however strongly expressed in these cells (Table 1).

\section{Discussion}

$\mathrm{HCC}$ is one of the most frequent and lethal cancers worldwide. Reliable markers for early diagnosis are still missing. We investigated ST6Gal I and sialoglycans for their expression and localization in liver tumors because hepatocytes are known to express ST6Gal I and produce sialylated plasma glycoproteins in large quantities. The development of liver diseases may be accompanied by a dysregulation of the sialoglycan metabolism. Therefore a comprehensive knowledge of ST6Gal I expression and that of sialoglycans in normal and cirrhotic liver and HCC is of interest for both basic and clinical research. We found both quantitative differences and differences in cellular localization of ST6Gal I and sialoglycan expression between normal liver and liver diseases.

In human normal liver, ST6Gal I was exclusively expressed in the Golgi region of hepatocytes but not in other cells such as biliary epithelial cells, endothelial cells, Kupffer cells, and Ito cells. Also in liver cirrhosis, hepatocytes are positive for ST6Gal I only in the Golgi region. The proliferating oval cells most likely representing stem cells and the pseudotubules were negative for ST6Gal I. This result indicates that ST6Gal I may play a role in liver cell differentiation and further supports the belief that the pseudotubules originate from the bile duct as demonstrated earlier by anticytokeratin mAb staining (Stosiek et al, 1985). ST6Gal I may be a suitable marker to distinguish hepatocellular from bile ductular cell lineage.

It has been reported that chronic ethanol intake down-regulates $\alpha 2,6$-sialyltransferase expression in rat liver (Rao and Lakshman, 1999). In the limited 
Table 1. Flow Cytometric and Immunocytochemical Analysis of ST6Gal I and Sialylated Glycan Expression on Human HCC Cell Lines HepG2 and HuH7

\begin{tabular}{|c|c|c|c|c|c|}
\hline \multirow[b]{2}{*}{ Target molecules } & \multirow[b]{2}{*}{ Reagents } & \multicolumn{2}{|c|}{ Flow cytometry } & \multicolumn{2}{|c|}{ Immunocytochemistry } \\
\hline & & HepG2 & HuH7 & HepG2 & HuH7 \\
\hline ST6Gal I & ST6-G1 & $-1+++^{a}$ & $-1+++^{a}$ & $\mathrm{G}+++$ & $\mathrm{G}+++$ \\
\hline$\alpha 2,6$-sialylated lactosamines & CD22Rg & +++ & +++ & $\mathrm{GM}+++$ & $\mathrm{GM}+++$ \\
\hline$\alpha 2,6$-sialylated oligosaccharides & SNA & +++ & +++ & $\mathrm{GM}+++$ & $\mathrm{GM}+++$ \\
\hline$\alpha 2,3$-sialylated oligosaccharides & $\mathrm{SnRg}$ & +++ & + & $\mathrm{GM}+++$ & $\mathrm{G}+++\mathrm{M}+$ \\
\hline$\alpha 2,3$-sialylated oligosaccharides & MAA & +++ & + & $\mathrm{GM}+++$ & $\mathrm{G}+++\mathrm{M}+$ \\
\hline TF & A78-G/A7 & - & + & - & GM+ \\
\hline Sialylated TF (pretreatment with VCN) & A78-G/A7 & +++ & +++ & $\mathrm{GM}+++$ & $\mathrm{GM}+++$ \\
\hline Tn & HB-Tn1 & - & - & - & - \\
\hline Sialosyl-Tn & HB-sTn1 & - & - & - & - \\
\hline Asialo-GM3 & Anti-asialo-GM3 & +++ & +++ & $\mathrm{GM}+++$ & $\mathrm{GM}+++$ \\
\hline GD3 & R24 & - & - & - & - \\
\hline 9-0-acetyl-GD3 & UM4D4 & $(+)$ & $(+)$ & - & - \\
\hline 7-0-acetyl-GD3 & U5 & + & + & GM+ & GM+ \\
\hline
\end{tabular}

a Pretreatment with saponin. - , negative for immunocytochemical staining/<1\% positive cells in flow cytometry; $(+), 1-5 \%$ positive cells;,$+ 5-30 \%$ positive cells;,$++ 30-60 \%$ positive cells;,$+++>60 \%$ positive cells. $\mathrm{G}=$ present in the Golgi region; $\mathrm{M}=$ present at the plasma membrane.

Table 2. Localization of ST6Gal I and Sialylated Ligands for CD22 and Sialoadhesin in Normal Liver Tissues and Various Liver Lesions

\begin{tabular}{|c|c|c|c|c|}
\hline Tissues & Cases & ST6-G1 & CD22Rg & SnRg \\
\hline Normal liver & 6 & & & \\
\hline Hepatocytes & & $\mathrm{G}+{ }^{a}$ & $\mathrm{~B}+$ & $\mathrm{B}+$ \\
\hline Bile ducts & & - & $\mathrm{M}+$ & $M+$ \\
\hline Kupffer cells & & - & $\mathrm{C}+$ & $\mathrm{C}+$ \\
\hline Endothelium & & - & $C+$ & $\mathrm{C}+$ \\
\hline Liver cirrhosis & 8 & & & \\
\hline Hepatocytes & & $\mathrm{G}+$ & $\mathrm{B}+$ & $\mathrm{B}+$ \\
\hline Oval cells & & - & $\mathrm{C}+$ & $\mathrm{C}+$ \\
\hline Well and moderately differentiated HCC & 6 & $\mathrm{G}+\mathrm{C}+$ & $\mathrm{G}+\mathrm{C}+\mathrm{M}+$ & $\mathrm{G}+\mathrm{C}+\mathrm{M}+$ \\
\hline Poorly differentiated HCC & 4 & $\mathrm{C}+1-$ & $\mathrm{C}+\mathrm{l}-$ & $\mathrm{C}+\mathrm{I}-$ \\
\hline Cholangiocarcinoma & 1 & - & $\mathrm{C}+\mathrm{M}+$ & $\mathrm{C}+\mathrm{M}+$ \\
\hline Malignant epitheloid & & & & \\
\hline Hemangioendothelioma & 1 & - & $\mathrm{C}+\mathrm{M}+$ & $\mathrm{C}+\mathrm{M}+$ \\
\hline
\end{tabular}

$\mathrm{B}=$ present along the bile canaliculi; $\mathrm{C}=$ present in the diffuse cytoplasm.

Scoring:,$+>30 \%$ positive cells; $+/-, 5-30 \%$ positive cells; - , cells not reactive.

${ }^{a}$ All cases in each group display similar staining patterns.

number of human alcoholic liver cirrhosis we investigated (four cases), we did not find significantly decreased expression of ST6Gal I in alcoholic liver cirrhosis in comparison with normal donor livers and posthepatitic liver cirrhosis.

Altered expression of ST6Gal I in benign and malignant hepatocellular lesions as compared with normal hepatocytes was observed in our study. The following results were obtained. (1) All normal hepatocytes and benign lesions express ST6Gal I. In contrast, the expression of ST6Gal I within a given $\mathrm{HCC}$ is often heterogeneous, ranging from none to very high amounts per cell. (2) Although ST6Gal I is distributed in the Golgi region in normal hepatocytes and benign lesions, malignant hepatocyte lesions reveal diffuse cytoplasm or diffuse cytoplasm/membrane distribution. (3) In poorly differentiated HCCs, part of the tumor cells apparently have lost the ability to express ST6Gal I. These differences may be explained on the basis of tumor cell pleomorphism-simultaneous up-regulation and downregulation of ST6Gal I within a given tumor mass and abnormal intracellular transport and distribution of the protein. Abnormally distributed ST6Gal I observed as diffuse staining of cytoplasm or cytoplasm/membrane may be an indication for increased release of sialyltransferase into blood. It will be of interest to see whether ST6Gal I as a serologic marker has any potential for clinical application in early detection and/or determination of progression of HCC. The pleomorphic expression and apolar distribution of ST6Gal I may be a sign of malignant transformation of hepatocytes and may be used as an additional parameter for diagnosis of HCC in histopathology. However, a larger number of cases must be investigated to permit final conclusions. 


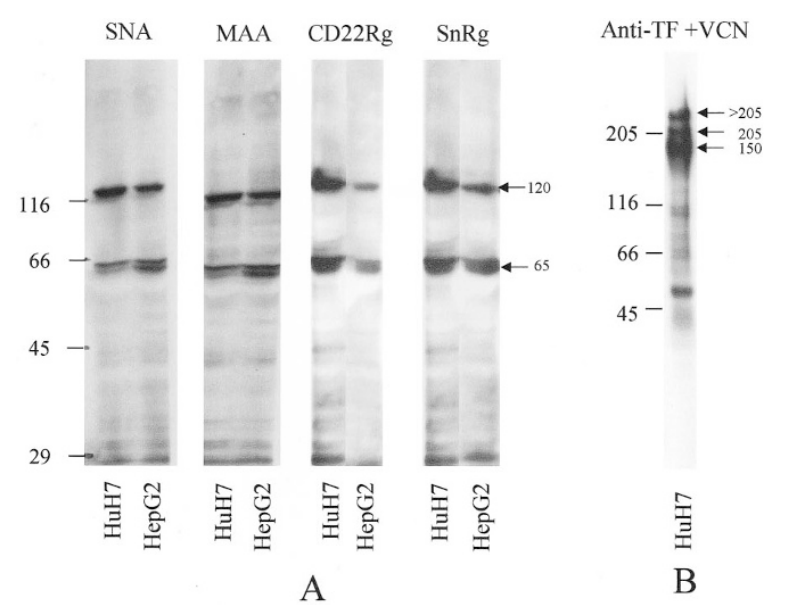

CD22Rg
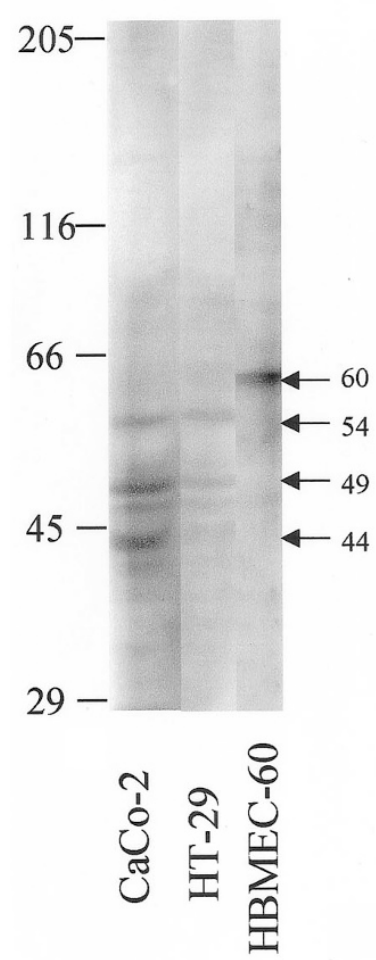

C

Figure 4.

A, Western blots of protein extracts from cell lines HuH7 and HepG2. Two major double bands (120 and $65 \mathrm{kDa}$ ) react with CD22Rg, SnRg, Sambucus nigra agglutinin (SNA), and Maackia amurensis agglutinin II (MAA) in both HCC cell lines. B, Immunoprecipitation of cell lysates from surface biotinylated $\mathrm{HuH} 7$ cells using anti-TF mAb and subsequent Western blotting revealed a series of TF-active glycoproteins with three main bands: $>205 \mathrm{kDa}, 205 \mathrm{kDa}$, and $150 \mathrm{kDa}$ in neuraminidase-treated $\mathrm{HuH} 7 . \mathrm{C}$, Western blots of protein extracts from cell lines HT-29, CaCo-2, and HBMEC-60. Three major bands $(54,49$, and $44 \mathrm{kDa})$ and one major band $(60 \mathrm{kDa})$ react with CD22Rg and $\mathrm{SnRg}$ for colonic epithelial cells and for endothelial cells, respectively.

An interesting finding was that ST6Gal I was present at the supranuclear cytoplasm surrounding the acinous and ductular structures in adenoid, pseudoglandular HCCs in comparison with the distribution of Golgi region surrounding the bile canaliculi in normal hepatocytes (Fig. 3G). It is tempting to speculate that these malignant hepatocytes show adenoid differentiation and that the acinous and ductular structures are formed by extension of bile canaliculi.

In contrast to ST6Gal I expression in all cases of HCC, one case each of cholangiocellular carcinoma and malignant epithelioid hemangioendothelioma did not express ST6Gal I. If our data, which must be categorized as a pilot study, are confirmed in further studies, ST6Gal I may be a suitable marker to distinguish $\mathrm{HCC}$ from other originating tumors such as cholangiocellular carcinoma and malignant endothelioma.

Sialylated glycans are recognized by members of the siglec family of lectins, such as CD22 recognizing Sia $\alpha 2,6 \mathrm{Gal} \beta 1,4 \mathrm{GlcNAc} \beta 1-\mathrm{R}$ residues and sialoadhesin reacting with $\alpha 2,3$-sialylated complex oligosaccharides (Hartnell et al, 2001; Sgroi et al, 1993). Binding sites of plant lectins such as SNA and MAA recognizing $\alpha$ 2,6-sialylated and $\alpha 2,3$-sialylated glycans in normal liver and HCC have been described in previous studies (Cao et al, 1996, 1999; Kaneko et al, 1995). In contrast to CD22 and sialoadhesin, these lectins may have a wider range of carbohydrate reactivities. Our study using the CD22Rg and SnRg may also point to sialoglycan ligands for these functionally relevant siglecs on nonimmune cells. Siglecs have been detected on tissue macrophages in liver tissue (Munday et al, 1999). Apparently, sialylation of tumor cells is a vital prerequisite for effective homing to and invasion of hepatic tissue (Petrick et al, 1994; Zhu et al, 2001).

In human normal liver and liver cirrhosis, hepatocytes and cholangiocytes express CD22 and sialoadhesin ligands at the apical membrane, along the bile canaliculi of hepatocytes, and at the luminal membrane of bile ducts. Kupffer cells, endothelial cells, and oval cells are positive for both ligands in the cytoplasm. The fact that these cells (found to be negative for ST6Gal I staining) nonetheless express $\alpha 2,6$ sialoglycans has also been observed by other groups (Kaneko et al, 1995; Dall'Olio et al, 2000). This could be explained by small amounts of active ST6Gal I enzyme below the threshold of $\mathrm{mAb}$ recognition, by an as yet unidentified $\alpha 2,6$-sialylating sialyltransferase, or by more complex mechanisms regulating $\alpha 2,6$ sialylation (Keppler et al, 1999).

Strong expression of CD22 and sialoadhesin ligands in the Golgi region, diffuse cytoplasm, and on the surface of HCC cells was demonstrated by immunocytochemical staining in cell lines and tissue sections and by FACS analysis. In poorly differentiated HCCs, most tumor cells also lose CD22 and sialoadhesin ligands. Cholangiocarcinoma and endothelial tumor strongly express CD22 and sialoadhesin ligands in the cytoplasm and plasma membrane. In normal and benign lesions, hepatocytes and cholangiocytes are most likely to be able to fully accomplish apical transport, whereby only CD22 and sialoadhesin ligands at the apical membrane can be visualized. Malignant lesions in which CD22 and sialoadhesin ligands are diffusely distributed seem to have lost the capacity for apical transport, resulting in accumulation of these glycoconjugates in the cytoplasm. We ob- 
served that cells with apolar distribution of CD22 and sialoadhesin ligands also showed an apolar arrangement of morphologic architecture. Apolar arrangement is regarded as a key step in the process of hepatocarcinogenesis (Stamatoglou and Hughes, 1994).

Rat hepatocytes express 9-O-acetylated sialic acids (Chammas et al, 1996). We found no mAb reaction with 9-O-acetylated disialoglycans in human hepatocytes or HCC. Instead, reactivity with an mAb against 7-O-acetyl-GD3 was observed at the cell surface and in the Golgi apparatus of human HCC cell lines. We further demonstrated that ligands for CD22, sialoadhesin, SNA, and MAA seemingly have identical major carrier glycoproteins in hepatocytes visualized as double bands of 65 and $120 \mathrm{kDa}$, respectively. Because both $\alpha 2,6$ - and $\alpha 2,3$ sialoglycan recognizing lectins identified the same glycoproteins, we assume that these proteins carry complex $\mathrm{N}$-linked oligosaccharides with terminal $\alpha 2,6$ and $\alpha 2,3$ sialylation.

These double bands may represent the most prominent sialoglycoproteins of the HCC cells; many more weaker bands as carriers for sialoglycans are observed as expected for liver cells. We have also noted that major carrier glycoproteins of ligands for CD22 and sialoadhesin are different in various cell types. In colonic epithelial cells, carrier proteins of ligands for CD22 and sialoadhesin are 54-, 49-, and 44-kDa glycoproteins. These sialoglycoproteins are unlike epithelial mucins, which are larger glycoproteins $(>200$ kDa), but like CD43 (Sikut et al, 1999). In endothelial cells, a $60-\mathrm{kDa}$ sialoglycoprotein is a major carrier protein of ligands for CD22 and sialoadhesin. The sialoglycoprotein may be involved in CD22-dependent homing of B cells on bone marrow (Nitschke et al, 1999). It will be of interest to further characterize the identity of these major sialoglycoproteins.

In a previous study, we showed that HCCs express TF and sialylated TF (Cao et al, 1999). In the present study, immunoprecipitation and Western blotting with the neuraminidase-treated cell line $\mathrm{HuH} 7$ revealed a series of TF-active glycoproteins with three main bands: >205 kDa, 205 kDa, and 150 kDa. Apparently carrier proteins of sialylated TF attached to O-linked oligosaccharides are different from the carrier proteins of ligands for CD22, SNA, sialoadhesin, and MAA.

Because ST6Gal I is a key enzyme for sialylation, many laboratories have measured its enzymatic activity in a wide spectrum of cells (Hubl et al, 2000). Enzymatic measurements may have several drawbacks, in particular for diagnostic purposes: (1) These methods are complex and require biochemical expertise. (2) Enzymatic activity may be unstable (ie, enzymes easily lose catalytic activity and for this reason only fresh tissue or cell samples can be used). In contrast, immunologic methods using mAbs are easily to perform, the enzyme retains its antigenicity even after storage of tissue samples, and more importantly for diagnostic value, immunologic methods are able to localize the enzyme as exemplified here. To our knowledge only one mAb reactive with ST6Gal I has been described so far (Gangopadhyay et al, 1998). In con- trast to our non-cross-reactive mAb STGal6, mAb 6B9 also reacted with two other liver proteins of approximate molecular weight of 65 and 100 kDa (Gangopadhyay et al, 1998). Thus, the new mAb against ST6Gal I, ST6-G1, is an appropriate antibody for basic research and clinical application.

In summary, our study demonstrated differential expression of ST6-G1 and sialoglycans in various tissues and in different situations. The clinical application of ST6Gal I as an immunohistologic marker in histopathology and possibly as a serum marker for early diagnosis of HCC will be investigated in further studies.

\section{Materials and Methods}

\section{Cell Lines and Cell Culture}

Human HCC cell lines HepG2 and HuH7 were grown in RPMI 1640 medium supplemented with 5\% FCS and DMEM supplemented with $10 \%$ FCS, respectively. Human colon carcinoma cell lines HT-29 and Caco-2 were cultured in RPMI 1640 medium and MEM, respectively; both media were supplemented with 10\% FCS. Human bone marrow endothelial cells (HBMEC-60; Rood et al, 2000), kindly provided by Dr. C. E. van der Schoot (University of Amsterdam, Amsterdam, the Netherlands), were maintained in endothelial cell culture medium supplemented with $20 \%$ FCS, $0.4 \%$ endothelial cell growth supplement/heparin, $0.1 \mathrm{ng} / \mathrm{ml}$ epidermal growth factor, $1 \mu \mathrm{g} / \mathrm{ml}$ hydrocortisone, $1 \mathrm{ng} / \mathrm{ml}$ fibroblast factor, $50 \mathrm{ng} / \mathrm{ml}$ amphotericin B, and $50 \mu \mathrm{g} / \mathrm{ml}$ gentamicin (PromoCell, Heidelberg, Germany).

\section{Tissue Specimens}

Tissue samples from 20 explanted and resected livers were studied. Pathologically altered tissues were taken from $10 \mathrm{HCCs}, 8$ cases of liver cirrhosis (4 posthepatitic, 4 alcoholic), 1 cholangiocellular carcinoma, and 1 malignant epithelioid hemangioendothelioma. Samples from six donor livers were used as normal tissues. All samples were immediately frozen and stored at $-80^{\circ} \mathrm{C}$. HCCs were diagnosed histologically and graded according to the classifications of the World Health Organization (Gibson and Sobin, 1978; Kojiro, 1997).

\section{Reagents}

The mAb against ST6Gal I was raised against a defined peptide sequence specific for ST6Gal I consisting of 16 amino acids (aa 121-137, NH2KYKVSYKGPGPGIKFS-COOH) of the human ST6Gal I (Grundmann et al, 1990). The peptide was coupled to $\mathrm{KLH}$ via an extra C-terminal cysteine. The mice were immunized sc with the peptide-KLH conjugate emulsified in complete Freund's adjuvant; multiple booster injections were applied ip without adjuvant. The last challenge was given 3 days before fusion. Standard fusion was done as described (Schwartz-Albiez et al, 1988). Hybridoma supernatants were screened for 
their specificity on solid-phase bound peptides in an ELISA using an anti-IgG mouse secondary antibody. Supernatants reactive with the specific peptide were further tested in Western blots on SDS-PAGE-separated commercially available sialyltransferases (ST3Gal III, recombinant rat $\alpha 2,3-\mathrm{N}$-sialyltransferase; ST3Gal I, recombinant rat $\alpha 2,3-\mathrm{O}$-sialyltransferase, both from Calbiochem, Bad Soden, Germany; ST6Gal I $\alpha 2,6-\mathrm{N}$-sialyltransferase derived from rat liver and purchased from Roche, Mannheim, Germany) and on tissue extracts of COS cells transfected with the ST6Gal I gene as described previously (Keppler et al, 1992). mAbs were purified from hybridoma supernatants by protein A-Sepharose 4B chromatography.

Soluble human CD22-immunoglobulin fusion protein (CD22Rg) and human sialoadhesin-immunoglobulin fusion protein $(\mathrm{SnRg})$ were used for studying expression and localization of CD22 and sialoadhesin ligands. CD22Rg contains the first three immunoglobulin-like domains of CD22 linked to the Fc portion of human IgG1 (Bergmann et al, 1998), and SnRg consists of the first 4 $\mathrm{N}$-terminal domains of the sialoadhesin fused to the Fc portion of human IgG1 (Hartnell et al, 2001). Fusion proteins were produced as described (Bergmann et al, 1998). In brief, cDNAs specific for CD22 and sialoadhesin were integrated into the immunoglobulin expression vector plg and were introduced into COS cells. CD22Rg and SnRg were purified from COS transfectant culture supernatants by chromatography on protein A-Sepharose 4B. For flow cytometry and Western blotting, CD22Rg and SnRg were preincubated with goat anti-human lgG1 (FC-specific) coupled to biotin in equimolar concentrations $(5 \mu \mathrm{m} / \mathrm{ml})$ for 1 hour at room temperature.

Two sialic acid-binding lectins of plant origin were used in the study: SNA recognizes the Neu5Ac $\alpha 2-$ 6Gal/GalNAc sequence (Shibuya et al, 1987); and MAA binds to sialylactosamine (NeuAc $\alpha 2-3 \mathrm{Gal} \beta 1$ 4GlcNAc $\beta$ ) (Imberty et al, 2000). Biotinylated SNA and MAA were obtained from Vector Laboratories (Burlingame, California).

The other mAbs used were as follows: UM4D4 (anti-9-O-acetyl-GD3; Vater et al, 1997), U5 (anti-7-Oacetyl-GD3; Kniep et al, 1995), and R24 (anti-GD3; Kniep et al, 1995) kindly supplied by Dr. C. Claus, University of Mainz; A78-G/A7 (anti-TF; Karsten et al, 1995) kindly provided by Dr. U. Karsten, MaxDelbrück Center, Berlin-Buch; and HB-Tn1 (anti-Tn; Dako, Copenhagen, Denmark) and HB-STn1 (anti-sTn; Dako).

\section{Immunocytochemistry and Affinity Cytochemistry}

To study the expression and localization of ST6Gal I as well as ligands for CD22 and sialoadhesin, cryostat sections (5- $\mu \mathrm{m}$ thickness) of livers were examined histochemically with anti- ST6Gal I, CD22Rg, and $\mathrm{SnRg}$. The cryostat sections were fixed with $-20^{\circ} \mathrm{C}$ acetone for 20 minutes. Then, the slides were treated with $3 \% \mathrm{H}_{2} \mathrm{O}_{2}$ in PBS for 30 minutes to block endogenous peroxidase and incubated with normal goat serum for 30 minutes to reduce nonspecific binding at room temperature. After washing with PBS, the slides were incubated with anti-ST6Gal I and CD22Rg as well as SnRg (both fusion proteins were preincubated with mouse anti-human IgG, at appropriate dilutions for 1 hour at room temperature). The thoroughly washed slides were treated with peroxidase-labeled goat anti-mouse immunoglobulin antiserum (Dako) for 30 minutes at room temperature. Color development during incubation with the peroxidase substrate diaminobenzidine was controlled under a microscope. Counterstaining was performed with hematoxylin. Negative controls were incubated with a comparable dilution of an isotype control (IgM or $\operatorname{lgG}$ ) of irrelevant specificity (Sigma, St. Louis, Missouri) instead of the $\mathrm{mAb}$ and the fusion proteins.

For obtaining information about cellular distribution of ST6Gal I and some glycan structures in established cell lines, cytochemical staining was performed with mAbs, biotinylated lectins, CD22Rg, or SnRg, as described above. Target cells were grown on sterile adhesion microscope slides for 1 day. At that time the medium was carefully aspirated, and the slides were air dried. Wrapped slides can be stored at $-80^{\circ} \mathrm{C}$ until use. Cells were fixed with $-20^{\circ} \mathrm{C}$ acetone for 20 minutes. Then, the slides were additionally treated with $0.2 \%$ saponin in PBS for 5 minutes. The possible glycolipid nature of carrier molecules was tested by fixation of tissue samples with $4 \%$ formalin for 5 minutes and immersion in methanol for 30 minutes at room temperature before the immunostaining was done as described above. For the detection of sialylated TF, cells were incubated with neuraminidase from Vibrio cholerae (VCN; Roche) at a concentration of $5 \mathrm{mU} / \mathrm{ml}$ in culture medium for 60 minutes at $37^{\circ} \mathrm{C}$ to remove $\alpha 2,3$-linked, $\alpha 2,6$-linked, or $\alpha 2,8$-linked sialic acid, washed, and than stained with anti-TF $\mathrm{mAb}$.

\section{Flow Cytometry}

The expression of ST6Gal I and some glycan structures in cell lines was determined semiquantitatively by FACS analysis. Cultured cells were stained at densities of $10^{6}$ cell/100 $\mu \mathrm{l}$ at $4^{\circ} \mathrm{C}$ for 15 minutes. Cells were first incubated with the appropriate primary $\mathrm{mAb}$, precomplexed CD22Rg or SnRg as described above or biotinylated lectins, followed by $(\mathrm{Fab})_{2}$ goat anti-mouse $\operatorname{lgG}$ and $\lg$ conjugated to FITC or streptavidin conjugated to FITC. After each incubation step, the cells were washed twice with PBS containing 1\% BSA. mAb W6/32 against MHC class I for positive control and an irrelevant mAb (HD20) for negative control were used in the same way. Cells were analyzed for immunofluorescence on a FACScan flow cytometer (Becton Dickinson, Mountain View, California), collecting data of 10,000 viable cells for each histogram. Dead cells were excluded by staining with VIA-PROBE (PharMingen, San Diego, California).

For intracellular staining with anti-ST6Gal I, cells were fixed with $3 \%$ fresh paraformaldehyde for 10 minutes and treated with $0.1 \%$ saponin in PBS for 30 
minutes at room temperature. Then the immunostaining was done as described above.

\section{SDS-PAGE}

Protein extracts of a rat liver and HepG2 as well as $\mathrm{HuH} 7$ were boiled in SDS-PAGE reducing buffer for 5 minutes and subsequently separated by SDS-PAGE and transferred to a polyvinylidene difluoride transfer membrane (Pall, Dreieich, Germany). The transfer membranes were incubated in $0.1 \%$ Tween 20 in PBS containing $5 \%$ nonfat dry milk overnight at $4^{\circ} \mathrm{C}$ and then incubated with anti-ST6Gal I, biotinylated SNA, MAA, or precomplexed CD22Rg or SnRg, for 1 hour at room temperature. The membranes were washed with $0.1 \%$ Tween 20 in PBS, followed by incubation with peroxidase-conjugated anti-mouse immunoglobulin or peroxidase-conjugated streptavidin for 1 hour at room temperature. After washing, the membranes were incubated with the Western Blot Chemiluminescence Reagent (NEN, Boston, Massachusetts) for 1 minute at room temperature. After draining excess detection reagent, the blots were exposed to x-ray film from 5 seconds to 5 minutes and then developed. Negative controls were incubated with a comparable dilution of an isotype control (lgM or $\lg G$ ) of irrelevant specificity (Sigma) instead of the $\mathrm{mAb}$ and the fusion proteins. For comparing carrier proteins of ligands for CD22 and sialoadhesin in various cell types, protein extracts of HT-29, CaCo-2, and HBMEC-60 were also analyzed by Western blotting using CD22Rg and SnRg as described above.

\section{Immunoprecipitation}

The Thomsen-Friedenreich oligosaccharide sequence (Gal $\beta 1-3 G a l N A c \alpha 1-O-)$ is a ubiquitous core structure found in a cryptic manner on many glycoproteins, predominantly masked by $\alpha 2,6$ - or $\alpha 2,3$-linked sialic acid. To examine whether sialylated TF and ligands for CD22 and sialoadhesin have similar carrier proteins in hepatocytes, carrier proteins of sialylated TF were also analyzed by immunoprecipitation and Western blotting. Biotinylation of surface proteins of VCN-treated $\mathrm{HuH} 7$ was performed using sulfosuccinimidobiotin (Pierce, Rockford, Illinois) as described elsewhere (Cao et al, 2001). Biotinylated surface proteins were immunoprecipitated using anti-TF mAb (A78-G/A7) coupled to protein A-Sepharose $4 \mathrm{~B}$ with a bridge rabbit anti-mouse immunoglobulin antibody. Precipitated biotinylated proteins were separated subsequently by PAGE using $8 \%$ acrylamide for separation gels and detected by peroxidase-conjugated streptavidin in Western blotting.

\section{Acknowledgements}

The authors thank Dr. G. Otto (Surgical Department, University of Heidelberg, Germany) and Prof. Banasch (DKFZ, German Cancer Research Centre) for providing the samples, Mr. Ditmar Greulich (DKFZ) for preparing tissues sections, and Prof. P. H. Krammer (DKFZ) for providing cell line HuH 7. We also thank Dr.
U. Karsten (Berlin, Germany) for providing monoclonal antibodies.

\section{References}

Bergmann M, Gross H-J, Abdelatty F, Möller P, Jaeken J, and Schwartz-Albiez R (1998). Abnormal surface expression of sialoglycans on B lymphocyte cell lines from patients with carbohydrate deficient glycoprotein syndrome I A (CDGS IA). Glycobiology 8:963-972.

Cao Y, Karsten U, Otto G, and Bannasch P (1999). Expression of MUC1, Thomsen-Friedenreich antigen, Tn, sialosyl$\mathrm{Tn}$, and $\alpha 2,6$-linked sialic acid in hepatocellular carcinomas and preneoplastic hepatocellular lesions. Virchows Arch 434: 503-509.

Cao Y, Merling A, Karsten U, and Schwartz-Albiez R (2001). The fucosylated histo-blood group antigens $\mathrm{H}$ type 2 (blood group O, CD173) and Lewis Y (CD174) are expressed on $\mathrm{CD}^{+} 4^{+}$hematopoietic progenitors but absent on mature lymphocytes. Glycobiology 11:677-683.

Cao Y, Stosiek P, Springer GF, and Karsten U (1996). Thomsen-Friedenreich-related carbohydrate antigens in normal adult human tissues: A systematic and comparative study. Histochem Cell Biol 106:197-207.

Chammas R, McCaffery JM, Klein A, Ito Y, Saucan L, Palade G, Farquhar MG, and Varki A (1996). Uptake and incorporation of an epitope-tagged sialic acid donor into intact rat liver Golgi compartments: Functional localization of sialyltransferase overlaps with $\beta$-galactosyltransferase but not with sialic acid O-acetyltransferase. Mol Biol Cell 7:1691-1707.

Crocker PR and Varki A (2001). Siglecs in the immune system. Immunology 103:137-145.

Dalziel M, Lemaire S, Ewing J, Kobayashi L, and Lau JT (1999). Hepatic acute phase induction of murine betagalactoside alpha 2,6 sialyltransferase (ST6Gal I) is IL-6 dependent and mediated by elevation of exon $\mathrm{H}$-containing class of transcripts. Glycobiology 9:1003-1008.

Dall'Olio F, Chiricolo M, Ceccarelli C, Minni F, Marrano D, and Santini $D$ (2000). $\beta$-galactoside $\alpha 2,6$-sialyltransferase in human colon cancer: Contribution of multiple transcripts to regulation of enzyme activity and reactivity with sambucus nigra agglutinin. Int $\mathrm{J}$ Cancer 88:58-65.

Gangopadhyay A, Perera S, and Thomas P (1998). Differential expression of $\alpha 2,6$-sialyltransferase in colon tumors recognized by a monoclonal antibody. Hybridoma 17:117-123.

Gibson JB and Sobin LH (1978). Histological typing of tumors of the liver, biliary tract and pancreas. International Histological Classification of Tumors, no. 20. Geneva: World Health Organization.

Gross H-J, Merling A, Moldenhauer G, and Schwartz-Albiez R (1996). Ecto- sialyltransferase of human B lymphocytes reconstitutes differentiation markers in the presence of exogenous CMP-N-acetyl-neuraminic acid. Blood 87:51135126 .

Grundmann U, Nerlich C, Rein T, and Zettlmeissl G (1990). Complete cDNA sequence encoding human $\beta$-galactoside $\alpha 2,6$-sialyltransferase. Nucleic Acids Res 18:667.

Hartnell A, Steel J, Turley H, Jones M, Jackson DG, and Crocker PR (2001). Characterization of human sialoadhesin, a sialic acid binding receptor expressed by resident and inflammatory macrophage populations. Blood 97:288-296. 
Harvey BE, Toth CA, Wagner HE, Steele GD Jr, and Thomas P (1992). Sialyltransferase activity and hepatic tumor growth in a nude mouse model of colorectal cancer metastases. Cancer Res 52:1775-1779.

Hubl U, Slim GC, and Zubkova OV (2000). Thin-layer chromatography and polyacrylamide gel electrophoresis-based assays for sialyltransferases using tetramethylrhodaminelabeled acceptors. Anal Biochem 285:92-99.

Imberty A, Gautier C, Lescar J, Perez S, Wyns L, and Loris R (2000). An unusual carbohydrate binding site revealed by the structures of two Maackia amurensis lectins complexed with sialic acid-containing oligosaccharides. J Biol Chem 275: 17541-17548.

Jain N, Sudhakar C, and Das MR (1993). Regulation of expression of $\beta$-galactoside $\alpha 2,6$-sialyltransferase in a rat tumor, Zajdela ascitic hepatoma. FEBS Lett 317:147-151.

Kaneko Y, Yamamoto H, Coliey KJ, and Moskal JR (1995). Expression of $\mathrm{Gal} \beta 1,4 \mathrm{GlcNAc} \alpha 2,6$-sialyltransferase and $\alpha 2,6$-linked sialoglycoconjugates in normal human and rat tissues. J Histochem Cytochem 43:955-963.

Karsten U, Butschak G, Cao Y, Goletz S, and Hanisch FG (1995). A new monoclonal antibody (A78G/A7) to the Thomsen-Friedenreich pan-tumor antigen. Hybridoma 14: 37-44.

Keppler OT, Hinderlich S, Langner J, Schwartz-Albiez R, Reutter W, and Pawlita M (1999). UDP-GIcNAc 2-epimerase: A regulator of cell surface sialylation. Science 284(5418): 1372-1376.

Keppler OT, Moldenhauer G, Oppenlander M, SchwartzAlbiez R, Berger EG, Funderud S, and Pawlita M (1992). Human Golgi beta-galactoside alpha-2,6-sialyltransferase generates a group of sialylated $\mathrm{B}$ lymphocyte differentiation antigens. Eur J Immunol 22:2777-2781.

Kitazume S, Tachida Y, Oka R, Shirotani K, Saido TC, and Hashimoto Y (2001). Alzheimer's beta-secretase, beta-site amyloid precursor protein-cleaving enzyme, is responsible for cleavage secretion of a Golgi-resident sialyltransferase. Proc Natl Acad Sci USA 98:13554-13559.

Kniep B, Claus C, Peter-Katalinic J, Dippold DAW, and Nimtz $M$ (1995). 7-O-acetyl-GD3 in human T-lymphocytes is detected by a specific T-cell-activating monoclonal antibody. J Biol Chem 270:30173-30180.

Kojiro M (1997). Pathology of hepatocellular carcinoma. In: Okuda $\mathrm{K}$ and Tabor E, editors. Liver cancer. New York: Churchill Livingstone, 165-187.

Munday J, Floyd H, and Crocker PR (1999). Sialic acid binding receptors (siglecs) expressed by macrophages. $\mathrm{J}$ Leukoc Biol 66:705-711.

Nitschke L, Floyd H, Ferguson DJP, and Crocker PR (1999). Identification of CD22 ligands on bone marrow sinusoidal endothelium implicated in CD22-dependent homing of recirculating B cells. J Exp Med 189:1513-1518.

Petrick AT, Meterissian S, Steele G Jr, and Thomas P (1994). Desialylation of metastatic human colorectal carcinoma cells facilitates binding to Kupffer cells. Clin Exp Metastasis 12: 108-116.

Pousset D, Piller V, Bureaud N, Monsigny M, and Piller F (1997). Increased $\alpha 2,6$-sialylation of $\mathrm{N}$-glycans in a transgenic mouse model of hepatocellular carcinoma. Cancer Res 57:4249-4256.
Rao MN and Lakshman MR (1999). Chronic ethanol consumption leads to destabilization of rat liver $\beta$-galactoside $\alpha 2,6$-sialyltransferase mRNA. Metabolism 48:797-803.

Rood PML, Calafat J, Von dem Borne AE, Gerritsen WR, and van der Schoot CE (2000). Immortalisation of human bone marrow endothelial cells: Characterisation of new cell lines. Eur J Clin Invest 30:618-629.

Roth J, Taatjes DJ, Lucocq JM, Weinstein J, and Paulson JC (1985). Demonstration of an extensive trans-tubular network continuous with the Golgi apparatus stack that may function in glycosylation. Cell 43:287-295.

Schneider F, Kemmner W, Haensch W, Franke G, Gretschel S, Karsten U, and Schlag PM (2001). Overexpression of sialyltransferase CMP-sialic acid:Gal beta 1-3GaLNAc-R alpha6-sialyltransferase is related to poor patient survival in human colorectal carcinomas. Cancer Res 61:4605-4611.

Schwartz-Albiez R, Dörken B, Hofmann W, and Moldenhauer G (1988). The B cell-associated CD37 antigen (gp40-52) structure and subcellular expression of an extensively glycosylated glycoprotein. J Immunol 140:905-914.

Sgroi D, Varki A, Braesch-Andersen S, and Stamenkovic I (1993). CD22, a B cell-specific immunoglobulin superfamily member, is a sialic acid-binding lectin. J Biol Chem 268: 7011-7018.

Shibuya N, Goldstein IJ, Broekaert WF, Nsimba-Lubaki M, Peeters B, and Peumans WJ (1987). The elderberry (Sambucus nigra L.) bark lectin recognizes the Neu5Ac(alpha 2-6)Gal/GalNAc sequence. J Biol Chem 262:1596-1601.

Sikut R, Andersson CX, Sikut A, Fernandez-Rodriguez J, Karlsson NG, and Hansson GC (1999). Detection of CD43 (leukosialin) in colon adenoma and adenocarcinoma by novel monoclonal antibodies against its intracellular domain. Int $\mathrm{J}$ Cancer 82:52-58.

Stamatoglou SC and Hughes RC (1994). Cell adhesion molecules in liver function and pattern formation. FASEB $J$ 8:420-427.

Stosiek P, Karsten U, and Kasper M (1985). Monoclonal anti cytokeratin antibodies reveal bile duct origin of cirrhotic ductular proliferations (dp). Virchows Arch A Pathol Anat Histopathol 407:233-235.

Tsuji S, Datta AK, and Paulson JC (1996). Systematic nomenclature for sialytransferases. Glycobiology 6:V-XIV.

Varki A (1999) Sialic acids. In: Varki A, Cummings R, Esko J, Freeze H, Hart G, and Marth J, editors. Essentials of glycobiology. Cold Spring Harbor, New York: Cold Spring Harbor Laboratory Press, 195-209.

Vater M, Kniep B, Gro $\beta$ H-J, Claus C, Dippold W, and Schwartz-Albiez R (1997). The 9-O-acetylated disialosyl carbohydrate sequence of CDw60 is a marker on activated human B lymphocytes. Immunol Lett 59:151-157.

Vierbuchen MJ, Fruechtnicht W, Brackrock S, Krause KT, and Zienkiewicz TJ (1995). Quantitative lectin-histochemical and immunohistochemical studies on the occurrence of alpha(2,3)- and alpha(2,6)-linked sialic acid residues in colorectal carcinomas: Relation to clinicopathologic features. Cancer 76:727-35.

Zhu Y, Srivatana U, Ullah A, Gagneja H, Berenson CS, and Lance P (2001). Suppression of a sialyltransferase by antisense DNA reduces invasiveness of human colon cancer cells in vitro. Biochim Biophys Acta 1536:148-160. 